\title{
Neutrophil to lymphocyte ratio as a predictor for diagnosis of early Periprosthetic joint infection
}

\author{
Bao-Zhan Yu' ${ }^{1,2}$, Jun Fu' ${ }^{1}$, Wei Chai ${ }^{1}$, Li-Bo Hao ${ }^{1 *}$ and Ji-Ying Chen ${ }^{1 *}$
}

\begin{abstract}
Background: Periprosthetic joint infection (PJI) is a catastrophic complication after total knee or hip arthroplasty. The diagnosis of $\mathrm{PJI}$ is very difficult, especially in the early postoperative period. The value of the neutrophil to lymphocyte ratio (NLR) is useful for diagnosing infectious diseases. The objective of this study was to investigate the accuracy of the NLR for the diagnosis of early PJI after total knee or hip arthroplasty.

Methods: We retrospectively evaluated consecutive primary total knee or hip arthroplasty and identified the patients who readmitted within the first 90 days postoperatively between January 2011 and October 2018.There were 20 cases diagnosed early PJI and 101 uninfected cases on the basis of the modified Musculoskeletal Infection Society (MSIS) criteria. The serum parameters including C-reactive protein (CRP), erythrocyte sedimentation rate (ESR), white blood-cell (WBC) count, NLR and interleukin-6 (IL-6) were compared between the two groups. Receiver operating characteristic curves were generated to estimate the optimal cutoff values for each parameter. The sensitivity, specificity, positive predictive value and negative predictive value for each parameter were calculated.

Results: The CRP, ESR, WBC, NLR and IL-6 values were all significally higher in the infected group than the uninfected group. The median of CRP was $66.6 \mathrm{mg} / \mathrm{l}$ in the infected group and $8.6 \mathrm{mg} / \mathrm{l}$ in the uninfected group $(p<0.001)$. The median of ESR was $34.8 \mathrm{~mm} / \mathrm{hr}$. in the infected group and $17.4 \mathrm{~mm} / \mathrm{hr}$. in the uninfected group $(p<0.001)$. In the infected group and uninfected group, the median of WBC was $8.2 \times 10^{9} / \mathrm{L}$ and $6.1 \times 10^{9} / \mathrm{L}(p=$ 0.002), respectively; while the median of NLR was 5.2 and $2.1(p<0.001)$. The median of IL-6 was $46 \mathrm{pg} / \mathrm{ml}$ and 6.4 $\mathrm{pg} / \mathrm{ml}(\mathrm{p}<0.001)$,respectively. The best parameter for the diagnosis of early PJI was IL-6 (AUC $=0.814$ ) followed by the NLR (AUC =0.802), CRP (AUC =0.793), ESR ( $A \cup C=0.744)$ and WBC ( $A \cup C=0.632)$.
\end{abstract}

Conclusions: This study is the first to show that NLR values are more accurate than CRP and may be considered as useful parameters for the diagnosis of early PJl because it is a cheap and convenient parameter to be calculated in daily practice without extra costs.

Keywords: Neutrophil to lymphocyte ratio, Early periprosthetic joint infection, C-reactive protein, Interleukin-6

\footnotetext{
*Correspondence: haolibo301@163.com; jiyingchen_301@163.com

'Department of Orthopaedics, Chinese People's Liberation Army General

Hospital, 28 Fuxing Road, Beijing 100853, Haidian District, China

Full list of author information is available at the end of the article
}

(c) The Author(s). 2020 Open Access This article is licensed under a Creative Commons Attribution 4.0 International License, which permits use, sharing, adaptation, distribution and reproduction in any medium or format, as long as you give appropriate credit to the original author(s) and the source, provide a link to the Creative Commons licence, and indicate if changes were made. The images or other third party material in this article are included in the article's Creative Commons licence, unless indicated otherwise in a credit line to the material. If material is not included in the article's Creative Commons licence and your intended use is not permitted by statutory regulation or exceeds the permitted use, you will need to obtain permission directly from the copyright holder. To view a copy of this licence, visit http://creativecommons.org/licenses/by/4.0/ The Creative Commons Public Domain Dedication waiver (http://creativecommons.org/publicdomain/zero/1.0/) applies to the data made available in this article, unless otherwise stated in a credit line to the data. 


\section{Background}

Periprosthetic joint infection (PJI) is a disastrous complication after total knee or hip arthroplasty with high morbidity and mortality. The early and accurate diagnosis of PJI is of great clinical significance for the treatment of PJI $[1,2]$. A less invasive procedure to retain the prosthetic components may be adopted in the early postoperative stage. However, the diagnosis of PJI is very difficult due to the lack of an absolute diagnostic test. It remains even more challenging for the diagnosis of early PJI because the normal periincisional swelling and erythema make it difficult to distinguish an early postoperative infection from the normal postoperative course.

It is essential to diagnose early PJI on the basis of a combination of clinical judgment, blood testing, synovial fluid aspiration, microbiologic and histopathologic inspections as well as imaging. There are various imaging techniques used in the assessment of PJI including radiographs, CT, MRI, ultrasound and nuclear medicine examinations. The ultrasonography (US) and MRI may be valuable tool for diagnosis of early PJI, which are characterized by soft tissue swelling, fluid collections and presence of sinus around the joint. However, there are very few literatures regarding for the diagnosis of early PJI because of lack of a suitable control group as the truly aseptic revisions are rare in the early postoperative period. Kim et al. [3] found that the serum CRP may be a wonderful screening test in the workup of early PII after TKA. However, Bedair et al. [4] reported that the sensitivity of CRP in serum for diagnosing early PJI was only $53 \%$. The synovial fluid aspiration is also an excellent test in the workup of early PJI. The sensitivity and specificity of synovial WBC counts are approximately $95-98.9 \%$ and $91-100 \%$ respectively [3-5]. But the synovial fluid aspiration is an invasive operation and sometimes it is very hard to obtain synovial fluid even though repeat joint aspiration, especially for the hip joint.

Among the various kinds of tests, it is very critical to find a simple and practical marker for diagnosing early PJI. The serum WBC count has very little utility due to the very low sensitivity as markers of PJI [6]. In recently, the value of the neutrophil to lymphocyte rate (NLR) that is obtained from the absolute neutrophil and absolute lymphocyte counts of a complete blood count is routinely used to predict outcomes as an available marker in oncology, cardiovascular diseases and infections [7-9]. Yombi et al. [10] found that the NLR may be potentially a better biomarker for the detection of early PJI after TKA because it had a faster normalization than CRP. However, further studies are still required to determine the conclusions through a comparative study. To our knowledge, there were no related studies. Therefore, the purpose of this study was to determine the accuracy of the NLR in the diagnosis of early PJI.

\section{Methods}

After Institutional Review Board approval for this study was obtained, we retrospectively reviewed the cases of consecutive primary total knee or hip arthroplasty in our institutions between January 2011 and October 2018. A total of 245 cases readmitted within the first 90 days postoperatively were identified. These patients were admitted to hospital again in 90 days for the following reasons: (1) staged bilateral total knee or hip arthroplasty, (2) dislocation, (3) patellar ligament rupture, (4) persistent wound drainage, (5) fever, (6) fracture, (7) erythema,(8) superficial infection, (9) postoperative infection was suspected.

Blood samples for CRP, ESR, IL-6 and other markers were taken on the morning after readmission for all patients and were then sent to the medical laboratory center for testing as soon as possible. All data including CRP, ESR, white blood cell (WBC) count, IL-6, neutrophil count and lymphocyte count were obtained from electronic medical records by manual chart review. The NLR was obtained as the absolute neutrophil count divided by the absolute lymphocyte count.

The aspirations were performed for these patients which resulted from symptoms such as persistent wound drainage,fever, erythema, superficial infection and postoperative infection being suspected. The synovial fluids were used for bacterial culture, a leukocyte esterase (LE) strip test and synovial fluid analysis (white blood-cell [WBC] count and polymorphonu-clear leukocyte [PMN] percentage).

These inflammatory arthritis, such as rheumatoid arthritis, ankylosing spondylitisetc were excluded in order to ruling out interference with other possible preconditions associated with elevated inflammatory markers. Also excluded were 1) Patients with superficial infection (24cases); 2) postoperative fever (12cases, because of upper-respiratory infection, urinary tract infection); 3) a history of malignancy (4cases); 4) missing critical data (including CRP,IL-6 or ESR).

These exclusions left 121 cases were analyzed. There were 20 infected cases and 101 uninfected according to the Musculoskeletal Infection Society (MSIS) criteria [11] (Table 1). Eighteen cases that satisfied one of two major criteria: a sinus tract communicating with the prosthesis (16), two positive periprosthetic cultures with phenotypically identical organisms (2); 2 cases that satisfied three of five minor criteria: a single positive intraoperative periprosthetic tissue culture, elevated synovial fluid white blood cell (WBC) count and a positive histological analysis of periprosthetic tissue (> 5 neutrophils per high power field) (Fig. 1).

There were 19 cases that underwent debridement, antibiotics and implant retention (DAIR) and one case was managed with one-stage revision in the infected group. Three cases in the infected group failed and no cases were diagnosed and reoperated for PJI in the uninfected 
Table 1 The Modified Musculoskeletal Infection Society (MSIS) criteria

\begin{tabular}{|c|c|c|c|}
\hline \multicolumn{4}{|c|}{ PJl is present if one of two major criteria or three of five minor criteria exists: } \\
\hline \multirow{2}{*}{$\begin{array}{l}\text { Major } \\
\text { criteria }\end{array}$} & \multicolumn{3}{|l|}{ 1. A communicating sinus tract with the prosthesis; or } \\
\hline & \multicolumn{3}{|l|}{$\begin{array}{l}\text { 2. Two positive periprosthetic cultures with phenotypically } \\
\text { identical organisms }\end{array}$} \\
\hline \multirow{6}{*}{$\begin{array}{l}\text { Minor } \\
\text { criteria }\end{array}$} & Having three of the following minor criteria: & Acute PJI (< 90 days) & Chronic PJI (> 90 days) \\
\hline & 1. Serum CRP or ESR & ESR: no threshold & ESR: $>30 \mathrm{~mm} / \mathrm{h}$ \\
\hline & 2. Synovial fluid WBC count or & 10,000 cells/ $\mu \mathrm{L}$ &  \\
\hline & Changes in leukocyte esterase test strip & + or ++ & + or ++ \\
\hline & 3. Synovial fluid PMN \% & $90 \%$ & $80 \%$ \\
\hline & 4. Positive histologic analysis of the periprosthetic tissue & $\begin{array}{l}>5 \text { neutrophils per high-power } \\
\text { field }(\times 400)\end{array}$ & $\begin{array}{l}>5 \text { neutrophils per high-power } \\
\text { field }(\times 400)\end{array}$ \\
\hline
\end{tabular}

ESR Erythrocyte sedimentation rate, CRP C-reactive protein, WBC White blood cell count, $P M N$ Neutrophils differential

group with at least 1-year follow-up. Twenty four cases of superficial infection were also analyzed. There was no case that were diagnosed and reoperated for PJI with at least 1-year follow-up.(Table 2).

\section{Statistical analysis}

The categorical variables were assessed using chi-square tests and parametric data were analyzed using $\mathrm{t}$ tests. The results of the diagnostic tests in the two groups were compared using the Mann-Whitney test. Statistical significance was considered as a $p<0.05$. The whole statistical analysis were performed with use of the statistical software packages R (The R Foundation) and Empower (X\&Y Solution). The Receiver operating characteristic (ROC) curves and area under the curve (AUC) were calculated to examine each parameter for the evaluation of early PJI. The Youden index was used to determine the optimal threshold for each parameter as a diagnostic tool for early PJI. The sensitivity and specificity of each parameter were calculated.

\section{Results}

There were 20 cases in infected group and 101 cases in uninfected group based on the MSIS criteria. There was no significantly different in age, time interval, BMI, joint (hip or knee) between two groups. The characteristics of each cohort are shown in (Table 3).

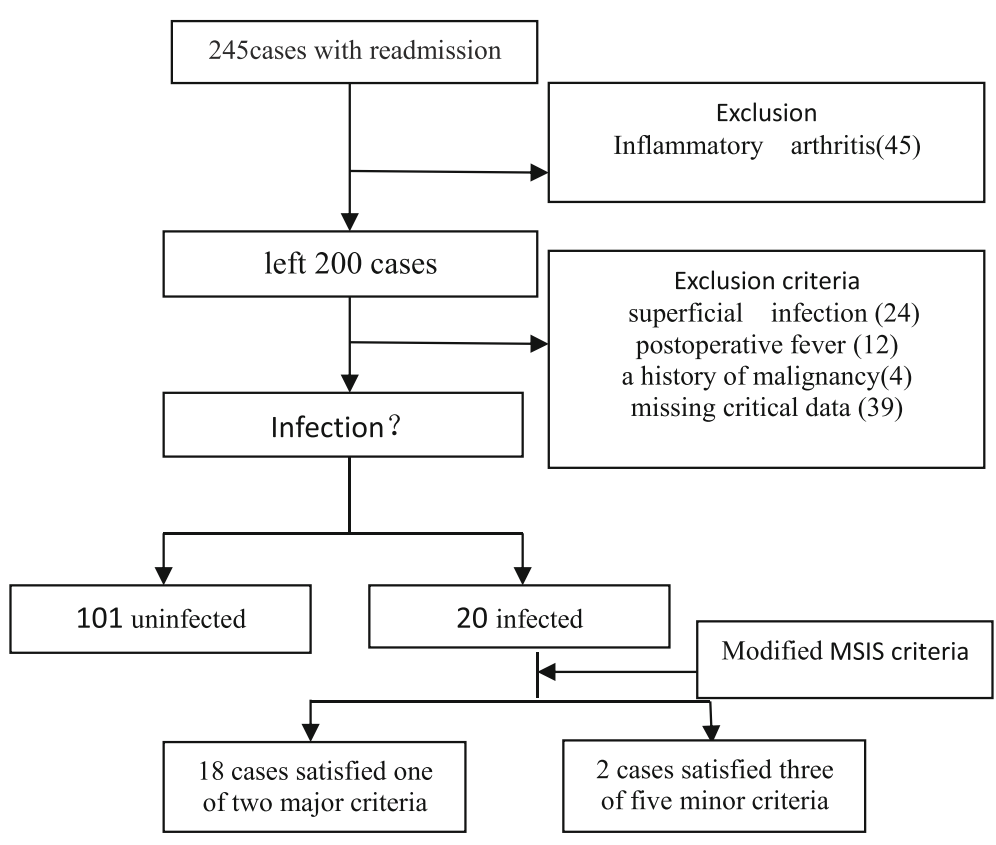

Fig. 1 Flowchart 
Table 2 Detailed characteristics of patients with superficial infection

\begin{tabular}{|c|c|c|c|c|c|}
\hline Patient & $\begin{array}{l}\text { Sex(male/M } \\
\text { female/F) }\end{array}$ & Joint (knee/hip) & Primary disease & Interval time readmission(day) & Follow-up time(months) \\
\hline 1 & $\mathrm{~F}$ & Knee & $\mathrm{OA}$ & 15 & 76 \\
\hline 2 & $\mathrm{~F}$ & Knee & $\mathrm{OA}$ & 30 & 71 \\
\hline 3 & $\mathrm{~F}$ & Knee & $\mathrm{OA}$ & 21 & 56 \\
\hline 4 & M & Knee & $\mathrm{OA}$ & 23 & 53 \\
\hline 5 & M & Knee & $\mathrm{OA}$ & 11 & 48 \\
\hline 6 & $\mathrm{~F}$ & Knee & $\mathrm{OA}$ & 7 & 35 \\
\hline 7 & $\mathrm{~F}$ & knee & $\mathrm{OA}$ & 45 & 32 \\
\hline 8 & $\mathrm{~F}$ & Knee & $\mathrm{OA}$ & 25 & 28 \\
\hline 9 & M & Hip & ONFH & 14 & 36 \\
\hline 10 & $\mathrm{~F}$ & Hip & ONFH & 8 & 27 \\
\hline 11 & M & Hip & ONFH & 5 & 24 \\
\hline 12 & M & Hip & ONFH & 14 & 24 \\
\hline 13 & $\mathrm{~F}$ & Knee & $\mathrm{OA}$ & 10 & 18 \\
\hline 14 & $\mathrm{~F}$ & Hip & $\mathrm{OA}$ & 11 & 29 \\
\hline 15 & $\mathrm{~F}$ & Hip & $\mathrm{DDH}$ & 9 & 31 \\
\hline 16 & M & Knee & $\mathrm{OA}$ & 7 & 24 \\
\hline 17 & M & Knee & $\mathrm{OA}$ & 15 & 25 \\
\hline 18 & $\mathrm{~F}$ & Hip & $\mathrm{OA}$ & 8 & 22 \\
\hline 19 & M & Hip & ONFH & 6 & 24 \\
\hline 20 & $\mathrm{~F}$ & Hip & $\mathrm{DDH}$ & 23 & 18 \\
\hline 21 & $\mathrm{~F}$ & Hip & $\mathrm{DDH}$ & 13 & 17 \\
\hline 22 & $\mathrm{~F}$ & Knee & $\mathrm{OA}$ & 17 & 15 \\
\hline 23 & $\mathrm{~F}$ & Knee & $\mathrm{OA}$ & 8 & 15 \\
\hline 24 & M & Knee & $\mathrm{OA}$ & 12 & 12 \\
\hline
\end{tabular}

OA Osteoarthritis, ONFH Osteonecrosis of the Femoral Head, DDH Developmental dysplasia hip

The median of CRP was $66.6 \mathrm{mg} / \mathrm{l}$ ((interquartile range [IQR], 0.5 to 208.9$)$ in the infected group and $8.6 \mathrm{mg} / \mathrm{l}$ ([IQR], 0.5 to 79.4$)$ in the uninfected group $(p<0.001)$. The median of ESR was $34.8 \mathrm{~mm} / \mathrm{hr}$. ([IQR], 7 to 78 ) in the infected group and $17.4 \mathrm{~mm} / \mathrm{hr}$. ([IQR], 3 to 79 ) in

Table 3 Patient characteristics

\begin{tabular}{llll}
\hline Variable & Infected $(\mathbf{n = 2 0})$ & Uninfected $(\mathbf{n = 1 0 1 )}$ & $\boldsymbol{P}$-value \\
\hline Sex & & & 0.03 \\
$\quad$ Male & $13(65 \%)$ & $22(21.78 \%)$ & \\
$\quad$ Female & $7(35 \%)$ & $79(78.22 \%)$ & \\
Age & $68.2(44-82)$ & $64.9(20-84)$ & 0.968 \\
BMl $\left(\mathrm{kg} / \mathrm{m}^{2}\right)$ & $28.43(18.37-48.68)$ & $27.74(18.59-38.35)$ & 0.734 \\
Joint & & & 0.562 \\
$\quad$ Hip & $3(15 \%)$ & $25(24.75 \%)$ & \\
$\quad$ Knee & $17(85 \%)$ & $76(75.25 \%)$ & \\
Time & $45(7-87)$ & $40(5-90)$ & 0.132 \\
\hline
\end{tabular}

BMI Body mass index the uninfected group $(\mathrm{p}<0.001)$. In the infected group and uninfected group, the median of $\mathrm{WBC}$ was $8.2 \times 10^{9}$ /L (IQR, 4.2 to $28.1 \times 10^{9} / \mathrm{L}$ ) and $6.1 \times 10^{9} / \mathrm{L}$ (IQR, 3.0 to $\left.14.8 \times 10^{9} / \mathrm{L}\right)(p=0.002)$, respectively; while the median of NLR was 5.2 ([IQR], 0.9 to 18.8 ) and 2.1([IQR], 0.8 to 6.4$)(p<0.001)$. The median of IL-6 was $46 \mathrm{pg} / \mathrm{ml}$ ([IQR], 1.5 to 347$)$ and $6.4 \mathrm{pg} / \mathrm{ml}$ ([IQR], 1.5 to 40.9 ) $(p<0.001)$, respectively. (Table 4$)$.

Table 4 Median values of diagnostic measures between infected and uninfected group

\begin{tabular}{llll}
\hline Variable & Infected $(\mathbf{n}=\mathbf{2 0})$ & Uninfected $(\mathbf{n}=\mathbf{1 0 1})$ & $P$-value \\
\hline CRP & $66.6(0.5-208.9)$ & $8.6(0.5-79.4)$ & $<0.001$ \\
ESR & $34.8(7.0-78.0)$ & $17.4(3.0-79.0)$ & $<0.001$ \\
WBC & $8.2(4.2-28.1)$ & $6.1(3.0-14.8)$ & 0.0024 \\
NLR & $5.2(0.9-18.8)$ & $2.1(0.8-6.4)$ & $<0.001$ \\
IL-6 & $46.0(1.5-347.0)$ & $6.4(1.5-40.9)$ & $<0.001$ \\
\hline CRP C-reactive protein, ESR Erythrocyte sedimentation rate, WBC White blood
\end{tabular}
cell count, IL-6 Interleukin-6, NLR Neutrophil-to-lymphocyte ratio 


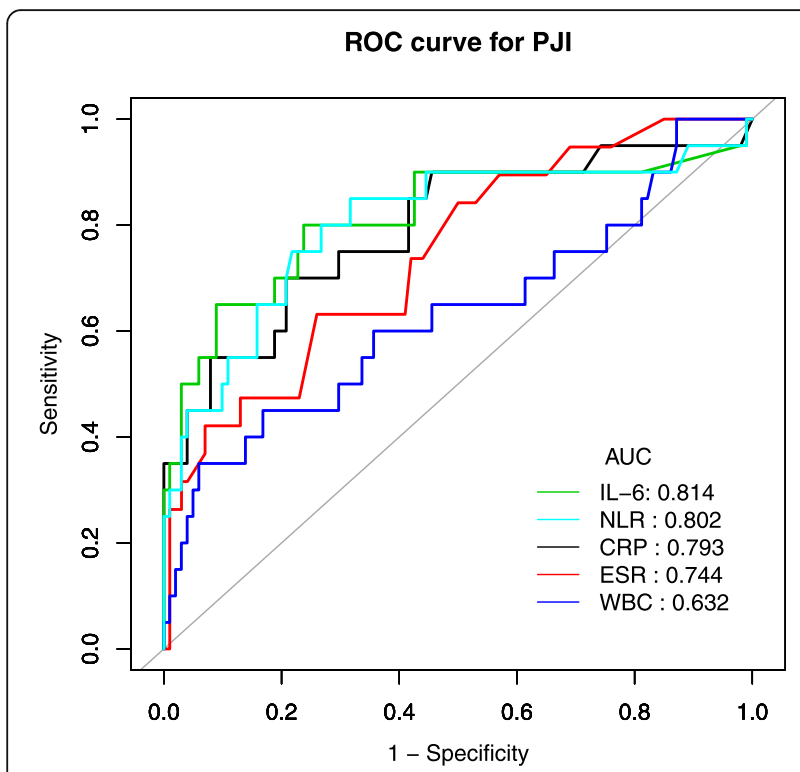

Fig. 2 The ROC curves for C-reactive protein (CRP), erythrocyte sedimentation rate (ESR), white blood-cell count (WBC), neutrophil to lymphocyte ratio (NLR) and interleukin-6(IL-6) in predicting early PJI

The ROC curve analysis showed the IL- 6 was the best test for diagnosing early PJI (AUC $=0.814$ ) followed by the NLR (AUC =0.802), CRP (AUC =0.793), ESR (AUC $=0.744)$ and $\mathrm{WBC}(\mathrm{AUC}=0.632)$ (Fig. 2). We identified the cutoff values for the IL- 6 at $8.07 \mathrm{pg} / \mathrm{ml}$. The sensitivity, specificity, positive predictive value (PPV), and negative predictive value (NPV) was 80, 76.2, 40, and 95.1\%, respectively. With the calculated cutoff value of NLR set at 2.13, the sensitivity, specificity, positive predictive value (PPV), and negative predictive value (NPV) was $85,68.3,34.7$, and $95.8 \%$, respectively (Table 5).

\section{Discussion}

It is very difficult to identify an early postoperative infection after total knee or hip arthroplasty because there is no an absolute diagnostic criteria. In this study, we determined interleukin-6(IL-6) in serum had the highest accuracy as a marker for diagnosing early PJI, followed by the NRL, the C-reactive protein, the erythrocyte sedimentation rate, and the white blood cell count.
Interleukin- 6 is an inflammatory cytokine produced by activated monocytes and macrophages and induces to product some major acute-phase proteins, including CRP [12]. Some studies have showed that the IL-6 in serum was a valuable marker for diagnosis of PJI. Di Cesare et al. [13] showed serum IL-6 was more accurate marker than either ESR or the CRP level for diagnosing chronic PJI. A meta-analysis [14] published that IL-6 in serum was a potential superior diagnostic test compared to the conventional CRP and ESR, with the pooled sensitivity and specificity of 97 and $91 \%$, respectively. At present there were no reports about IL-6 in serum as a useful marker for diagnosis of early PJI. Maniar et al. [15] found the normal trajectory of IL-6 had a more rapid increase and quicker return to normal than CRP after uncomplicated TKA. Although the author could not comment specifically on the value in diagnosing PJI, any deviation of IL-6 from a known normal trajectory could promptly make a decision to perform knee aspiration to diagnose early PJI. However, in our study, when we identified the cutoff values for the IL-6 at $8.07 \mathrm{pg} / \mathrm{ml}$, the sensitivity, specificity was $80,76.2 \%$, respectively. IL6 in serum may be a useful marker for diagnosing early PJI. This is the first to assess the use of serum IL- 6 to diagnose early PJI.

The serum IL- 6 was not often detected because of expensive costs. Interestingly, another indicator, NLR performed very well and had the high AUC (0.802). The accuracy of the value of NLR was less than IL- 6 but greater than CRP. It may be more suitable for screening to diagnose early PJI because it is convenient to be obtained in daily practice without extra costs.

The value of the NRL had been shown a significant association with infection. Dogruel et al. [16] demonstrated there were statistically significant correlations between NLR and prolonged hospital stay and postoperative antibiotic doses for the treatment of odontogenic infection. Josse et al. [17] reported that the values of NLR $\geq 2.3$ in the pre-operation may be an independent predictor for major operative complications such as wound infection in patients undergoing colorectal surgery. Bolat et al. [18] investigated the relationship between NLR and early postoperative infection as a complication of penile prosthesis implantation (PPI).

Table 5 Values of serum parameters in predicting PJI

\begin{tabular}{|c|c|c|c|c|c|c|c|}
\hline & AUC & (95\%CL) & Threshold & Sensitivity & Specificity & PPV & NPV \\
\hline CRP & 0.793 & $0.668-0.918$ & $9.27 \mathrm{mg} / \mathrm{l}$ & 0.700 & 0.792 & 0.400 & 0.930 \\
\hline ESR & 0.744 & $0.621-0.867$ & $22 \mathrm{~mm} / \mathrm{hr}$ & 0.632 & 0.740 & 0.316 & 0.914 \\
\hline WBC & 0.632 & $0.479-0.784$ & $8.91 \times 10^{9} / \mathrm{L}$ & 0.350 & 0.941 & 0.538 & 0.880 \\
\hline NLR & 0.802 & $0.674-0.930$ & 2.13 & 0.850 & 0.683 & 0.347 & 0.958 \\
\hline IL -6 & 0.814 & $0.683-0.945$ & $8.07 \mathrm{pg} / \mathrm{ml}$ & 0.800 & 0.762 & 0.400 & 0.951 \\
\hline
\end{tabular}

AUC Area of under the receiver operating characteristic curve, PPV Positive predictive value, NPV Negative predictive value 
And the values of NLR may be a valid laboratory predictor for early postoperative infectious complications for patients who went through PPI. De Jager et al. [19] described the NLR had the highest AUC of 0.73 and differed significantly from the CRP level of 0.62 . The NLR was better value in predicting bacteremia than routine parameters like CRP. Yombi et al. [10] reported that the NLR had a distribution trajectory in a standard postoperative period after TKA, and returned to normal values faster than CRP. It was potentially a better biomarker to follow post-operative inflammation or early infection after TKA. On the basis of our data, with the calculated threshold of NLR set at 2.13, the sensitivity, specificity, positive predictive value (PPV), and negative predictive value (NPV) was $85,68.3,34.7$, and $95.8 \%$, respectively. To our knowledge, this is the first study to assess the NLR in diagnosing early PJI.

Although there were some literatures that elaborate possible explanations about the relation between elevated NLR and the development of [20-22], it remained unclear about the exact mechanism. The neutrophils play pivotal roles in the progression of the bacterial infection. The key cell types of the innate immune system as well as the first cellular line of defense against infection are neutrophils. Lymphocytes are involved in adaptive immune response. The physiological immune responses of circulating leukocytes to various stressful events has distinctive feature: increase in neutrophil count and decrease in lymphocyte count. An increase in neutrophils is an inflammatory reaction, particularly when caused by a bacterial infection [23]. Lymphocytopenia has also been described as a diagnostic marker of bacterial infection [24]. Therefore, the NLR is thought to have stronger discriminative power for predicting bacteremia compared to discrimination based on neutrophilia or lymphocytopenia alone. The most crucial finding of this study was that NLR may be a useful parameter for diagnosing early PJI.

There are some limitations of this study that should be considered. First, this is retrospective study and the inherent limitations exist. There may be some selection bias because several potential cases were excluded due to no record of the serum ESR, CRP, or IL-6. Although this study is a retrospective design, it may provide some useful information for diagnosing early PJI. Second, there is no consensus on definition of the early PJI. We defined 90 days as early PJI and shorter time needed to be detected. Finally, we recruited 121 cases to the study. Although the total sample size was relatively sufficient, there was a small sample size in infected cases that limited the statistical power of our conclusions. Therefore, our findings should be confirmed in a larger study and at multiple institutions.

\section{Conclusions}

The findings of this study have shown that the IL-6 was the best test for diagnosis of early PJI. But it was not often detected because of expensive costs. However, the NLR that has the higher accuracy for the diagnosis of early PJI than CRP may be considered as a useful parameter for the diagnosis of early PJI because it is easy, cheap and convenient to be calculated in daily practice without extra costs. However, further studies evaluating the accuracy of NLR in more patients are needed to verify the findings of the present study.

\begin{abstract}
Abbreviations
PJl: Periprosthetic joint infection; NLR: Neutrophil to lymphocyte ratio; TKA: Total knee arthroplasty; AUC: The area under the ROC curve; BMl: Body mass index; CRP: Creactive protein; ESR: Erythrocyte sedimentation rate; LL-6: Interleukin-6; LE: Leukocyte esterase; MSIS: Musculoskeletal Infection Society; NPV: Negative predictive value; PMN\%: Percentage of polymorphonuclear cells; PPV: Positive predictive value; ROC: Receiver operating characteristic; WBC: White blood cell; PPI: Penile prosthesis implantation
\end{abstract}

\section{Acknowledgements}

None.

\section{Authors' contributions}

BZY performed data collection and data analysis. BZY and JF drafted the manuscript. BZY, WC, LBH and JYC conceived of the study, participated in the design of the study, performed data interpretation, and participated in coordination. The authors read and approved the final manuscript.

\section{Funding}

This work was supported by the grants of the Military Medical and Health Achievements Expansion Project (19WKS09).

\section{Availability of data and materials}

We do not wish to share our data, because some of the patient's data regarding individual privacy, and according to the policy of our hospital, the data could not be shared with others without permission. An anonymised form of the data could be made available from the corresponding author upon reasonable request.

\section{Ethics approval and consent to participate}

This study was approved by the Ethics Committee of the Chinese People's Liberation Army General Hospital and in accordance with the standards of the National Research Council. Written informed consent was obtained from all participants.

\section{Consent for publication}

Not applicable.

\section{Competing interests}

The authors declare that they have no competing interests.

\section{Author details \\ 'Department of Orthopaedics, Chinese People's Liberation Army General Hospital, 28 Fuxing Road, Beijing 100853, Haidian District, China. \\ ${ }^{2}$ Department of Orthopaedics, Bao Ding GemFlower Eastern Hospital, Bao Ding, Hebei, P. R. China.}

Received: 19 April 2020 Accepted: 5 October 2020

Published online: 27 October 2020

\section{References}

1. Whitehouse MR, Parry MC, Konan S, Duncan CP. Deep infection after hip arthroplasty: staying current with change. Bone Joint J. 2016;98-B:27-30.

2. George DA, Gant V, Haddad FS. The management of periprosthetic infections in the future: a review of new forms of treatment. Bone Joint J. 2015;97-B:1162-9. 
3. Kim S-G, Kim J-G, Jang K-M, Han S-B, Lim H-C, Bae J-H. Diagnostic value of synovial white blood cell count and serum C-reactive protein for acute Periprosthetic joint infection after knee Arthroplasty. J Arthroplasty. 2017;32:3724-8.

4. Bedair H, Ting N, Jacovides C, Saxena A, Moric M, Parvizi J, et al. The mark Coventry award: diagnosis of early postoperative TKA infection using synovial fluid analysis. Clin Orthop Relat Res. 2011;469:34-40.

5. Yi PH, Cross MB, Moric M, Sporer SM, Berger RA, Della Valle CJ. The 2013 frank Stinchfield award: diagnosis of infection in the early postoperative period after total hip arthroplasty. Clin Orthop Relat Res. 2014;472:424-9.

6. Friedrich MJ, Randau TM, Wimmer MD, Reichert B, Kuberra D, StoffelWagner B, et al. Lipopolysaccharide-binding protein: a valuable biomarker in the differentiation between periprosthetic joint infection and aseptic loosening? Int Orthop. 2014;38:2201-7.

7. Angkananard T, Anothaisintawee T, McEvoy M, Attia J, Thakkinstian A. Neutrophil lymphocyte ratio and cardiovascular disease risk: a systematic review and meta-analysis. Biomed Res Int. 2018;2018:2703518-8.

8. Lowsby R, Gomes C, Jarman I, Lisboa P, Nee PA, Vardhan M, et al. Neutrophil to lymphocyte count ratio as an early indicator of blood stream infection in the emergency department. Emerg Med J. 2015;32:531-4.

9. Boissier R, Campagna J, Branger N, Karsenty G, Lechevallier E. The prognostic value of the neutrophil-lymphocyte ratio in renal oncology: a review. Urol Oncol. 2017:35:135-41.

10. Yombi JC, Schwab PE, Thienpont E. Neutrophil-to-lymphocyte ratio (NLR) distribution shows a better kinetic pattern than C-reactive protein distribution for the follow-up of early inflammation after total knee arthroplasty. Knee Surg Sports Traumatol Arthrosc. 2016;24:3287-92.

11. Panvizi J, Gehrke T, International Consensus Group on Periprosthetic Joint Infection. Definition of periprosthetic joint infection. J Arthroplasty. 2014;29:1331

12. Mihara M, Hashizume M, Yoshida H, Suzuki M, Shiina M. IL-6/IL-6 receptor system and its role in physiological and pathological conditions. Clin Sci (Lond). 2012;122:143-59.

13. Di Cesare PE, Chang E, Preston CF, Liu C. Serum interleukin-6 as a marker of periprosthetic infection following total hip and knee arthroplasty. J Bone Joint Surg Am. 2005;87:1921-7.

14. Berbari E, Mabry T, Tsaras G, Spangehl M, Erwin PJ, Murad MH, et al, Inflammatory blood laboratory levels as markers of prosthetic joint infection: a systematic review and meta-analysis. J Bone Joint Surg Am. 2010;92:2102-9.

15. Maniar RN, Navaneedhan G, Ranvir S, Maniar AR, Dhiman A, Agrawal A. What is the Normal trajectory of Interleukin-6 and C-reactive protein in the hours and days immediately after TKA? Clin Orthop Relat Res. 2019;477:41-6.

16. Dogruel F, Gonen Z-B, Gunay-Canpolat D, Zararsiz G, Alkan A. The neutrophil-to-lymphocyte ratio as a marker of recovery status in patients with severe dental infection. Med Oral Patol Oral Cir Bucal. 2017;22:e440-5.

17. Josse JM, Cleghorn MC, Ramji KM, Jiang H, Elnahas A, Jackson TD, et al. The neutrophil-to-lymphocyte ratio predicts major perioperative complications in patients undergoing colorectal surgery. Colorectal Dis. 2016;18:0236-42.

18. Bolat D, Topcu YK, Aydogdu O, Minareci S, Dincel C. Neutrophil to lymphocyte ratio as a predictor of early penile prosthesis implant infection. Int Urol Nephrol. 2017;49:947-53

19. de Jager CPC, van Wijk PTL, Mathoera RB, de Jongh-Leuvenink J, van der Poll T, Wever PC. Lymphocytopenia and neutrophil-lymphocyte count ratio predict bacteremia better than conventional infection markers in an emergency care unit. Crit Care BioMed Central. 2010;14:R192.

20. Shau HY, Kim A. Suppression of lymphokine-activated killer induction by neutrophils. J Immunol. 1988;141:4395-402.

21. Djeu JY, Serbousek D, Blanchard DK. Release of tumor necrosis factor by human polymorphonuclear leukocytes. Blood. 1990;76:1405-9.

22. Dindo D, Demartines N, Clavien P-A. Classification of surgical complications: a new proposal with evaluation in a cohort of 6336 patients and results of a survey. Ann Surg. 2004;240:205-13.

23. Zahorec R. Ratio of neutrophil to lymphocyte counts--rapid and simple parameter of systemic inflammation and stress in critically ill. Bratisl Lek Listy. 2001;102:5-14.

24. Wyllie DH, Bowler IC, Peto TE. Relation between lymphopenia and bacteraemia in UK adults with medical emergencies. J Clin Pathol. 2004;57:950-5.

\section{Publisher's Note}

Springer Nature remains neutral with regard to jurisdictional claims in published maps and institutional affiliations.

Ready to submit your research? Choose BMC and benefit from:

- fast, convenient online submission

- thorough peer review by experienced researchers in your field

- rapid publication on acceptance

- support for research data, including large and complex data types

- gold Open Access which fosters wider collaboration and increased citations

- maximum visibility for your research: over $100 \mathrm{M}$ website views per year

At BMC, research is always in progress.

Learn more biomedcentral.com/submissions 